\title{
Engineering an HL7 Interface and Wireless Infrastructure to Improve the Efficiency of ECG Analysis at Hartford Hospital
}

\author{
Danielle C. McGeary, Raymond R. Renouf, MSBME, Quing Zhu, Ph.D., John D. Enderle, Ph.D. \\ University of Connecticut, Department of Biomedical Engineering \\ 260 Glenbrook Road U-2247 \\ Storrs, CT 06269-2247
}

\begin{abstract}
This paper describes the integration of Hartford Hospital's current 12-lead electrocardiogram (ECG) software with the hospital's information systems (admit, discharge, transfer (ADT); orders; and billing) by engineering a Health Level Seven Standard Interface (HL7) specifically for the hospital's unique software amalgamation. Data formats for the wireless transmission of information were investigated. Distinctive requirements, work-flow, and validation procedures for interfacing the 12-lead ECG system using the HL7 standard were configured. Lastly, the implementation of a wireless transmission component was also developed so completed ECGs could be instantaneously sent to the ECG software directly from the ECG machine, where the analysis was performed. Spectrum analysis was utilized to scrutinize the hospital's current unique wireless infrastructure.
\end{abstract}

I. INTRODUCTION

Hartford Hospital currently uses General Electric's (GE) Marquette Universal System for Electrocardiography (MUSE) software for electrocardiogram (ECG) analysis and acquisition. While the software, as configured, was effective for physicians reviewing a patient's ECG waves, the system, as it was, had no way of interfacing with the hospital's ordering software, Sunrise Clinical Manager (SCM). With this lack of communication between SCM and MUSE, many physicians have been reading, confirming, and processing ECGs for billing that have no associated order.

This current workflow has created many concerns at Hartford Hospital in regards to reimbursement and especially compliance. ECGs that are processed without an order are lacking pivotal information such as a clinical indicator and the prescriber who placed the order. Without this information, reimbursement companies, such as Medicare and Medicaid, refuse to reimburse the hospital. This results in hundreds of thousands of dollars of revenue lost annually.

In order to ameliorate this problem, a Health Level Seven Standard Interface (HL7) was implemented into Hartford Hospital's current ECG system. [1] The HL7 interface brings together the hospital's existing orders, ADT, and results and billing software. Upon completion of this integration, every ECG taken in the hospital can be effortlessly associated with an order when an ECG is performed electronically from the ECG device. All of the ECG machines will be made wireless.

Upon completion of this project, all ECG orders entered in SCM will be transmitted via the HL7 server to the MUSE software. With this wireless connection to the MUSE server, an ECG order can be selected on the mobile ECG machine and associated with the patient. When the ECG is completed, it can be transmitted wirelessly back to the MUSE software with the associated order attached. The ECG can then be reviewed and resulted by a physician with all the appropriate demographics corresponding to the patient. The resulted ECG will then be sent back to SCM and attached to the patient's permanent electronic record and will also be sent for reimbursement.

This implementation requires much validation and is intrinsically unique to Hartford Hospital. A spectrum analyzer was used to study all areas in the hospital for wireless interference within a narrow $2.4 \mathrm{GHz}$ band. This is a cumbersome project that not only alters nursing workflow but also requires extensive networking and server engineering.

\section{METHODS}

A. Wireless Implementation and Testing

Silex Serial Bridges were chosen to be the wireless bridges used on all of the ECG machines. A mutually exclusive SSID, naming convention, and IP scheme was established for all the Silex Serial Bridges that were placed on the ECG carts. A unique, HIPAA compliant WPSK with Wi-Fi Protected Access (WPA) encryption was also established for this SSID.

Once a bridge was configured, a test ECG machine was defined and the wireless consistency and dependability was checked hospital wide. A spectrum analyzer was also used throughout to the hospital to look for any interference in the $2.4 \mathrm{GHz}$ band. As shown in Fig. 1, the Emergency Department (ED) as well as areas with telemetry were heavily analyzed.[2]

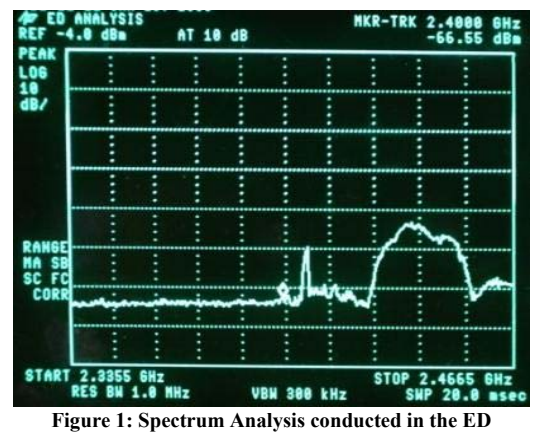




\section{B. HL7 Design and Testing}

First, the HL7 code on Hartford Hospital's main interface box, SeeBeyond, was developed so that all ECG orders would be directed to MUSE. While the HL7 protocol has a known structure with synonymous data segments and data fields, the information format of these messages may fluctuate between software systems. In order to ensure that SCM and MUSE could properly communicate and decipher message content by placing data in the appropriate fields of each software program, a validation plan was created.

In the validation plan, all ADT information was analyzed. This includes the patient identification (PID) segment, the patient visit segment (PV1), the event type segment (ETS), and the merge patient information segment (MRG). A second validation test was created for the orders component from SCM to MUSE. This specifically focused on the common order segment (ORC). Finally, a third validation plan was created for the results component (MUSE back to SCM) and the billing information file. This final validation honed in on the observation request segment (OBR), the observation result segment (OBX), and the financial transaction segment (FT1).

All of these segments were validated by tracing a myriad of ADT messages to MUSE and examining the outbound HL7 code from SCM and the inbound HL7 code to MUSE. If the ADT was not formatted properly in MUSE, the HL7 code would be altered until appropriate. All possible ECG order sets under various conditions were also followed from SCM to MUSE and then reviewed in the application. ECGs that were wirelessly transmitted from the ECG machine with an order to MUSE were reviewed. Finally, multiple ECGs were resulted and it was verified that all data in a patient's medical record in SCM appeared as desired.

\section{RESULTS}

For the wireless testing conducted throughout the hospital, very little interference was found. The spectrum analysis graphs also verified this. A laptop was also set up to continuously ping the IP address of the bridge as it was moving about the hospital on the ECG test machine. Almost all ping requests received an instantaneous response back from the bridge that was being used for the analysis.

The HL7 testing revealed multiple formatting errors in the HL7 code. SCM reported errors when initially receiving the resulted ECG from MUSE. The HL7 code was reformatted until SCM accepted the resulting message composition. Once SCM successfully accepted a result message, the appropriate outgoing HL7 message format in MUSE could be altered. This was done until all other HL7 segments were validated.

\section{DISCUSSION}

The theory of operation of this new system is that the Centricity Clinical Gateway (CCG), which essentially functions as a mailbox for MUSE, receives inbound ADT and Order (ORM) messages from external systems. In return, the CCG sends the outbound Results (ORU) and Billing (DFT) messages from the MUSE server to the external systems.
Essentially, no HL7 messages can enter or leave the MUSE server without first passing through the CCG.

When HL7 ECG order messages first leave SCM, they are passed to Hartford Hospital's main HL7 interface, SeeBeyond. Here, all HL7 messages are sorted and sent to their respective destination via a defined port and IP address. In this case, all ECG orders from SCM are sorted and sent to the CCG for MUSE. MUSE then constantly checks the CCG for new HL7 ECG order messages and collects them via a TCP/IP socket based communication protocol. [3] Once the MUSE server collects an ECG order it is then available to be used at an ECG machine.

Since this new system relies heavily on ECG orders from SCM, it was important to review all ECG order sets available in SCM during this implementation process. Since part of the ECG documentation problem involved a lack of clinical indication for performing the ECG, all order sets in SCM were revised to include this information.

The chest pain protocol, which is frequently ordered in the ED and cardiac intensive care units (ICUs), was also reviewed and revised. The chest pain protocol required that three ECGs be taken at the onset of chest pain, during chest pain, and post chest pain. The problem with this order set was that all three ECGs were combined into one order. This created a problem since MUSE requires a mutually exclusive order for each ECG performed. With the permission of the clinical staff, the chest pain protocol was programmed in SCM to now release three ECG orders every time the protocol is ordered.

\section{CONCLUSION}

The implementation of the HL7 interface and wireless infrastructure was a very unique and challenging integration for Hartford Hospital. Due to the unique wireless infrastructure and the multitude of diverse medical modalities that share the existing network, much testing and troubleshooting were required. The distinctive software programs that were joined via the HL7 code design were exclusive to Hartford Hospital. The extensive planning, design and validation procedures were necessary to discern flaws in the initial planning and design process and greatly supported and confirmed the engineering of the final ECG data flow.

\section{ACKNOWLEDGMENTS}

Sincere thanks to the Department of Biomedical Engineering, the Information Services Department, and the Department of Cardiology at Hartford Hospital and the Biomedical Engineering Department at the University of Connecticut.

\section{REFERENCES}

[1] ANSI Accredited Standards Developer, "Health Level Seven Implementation Support Guide," Health Level Seven Inc. Ann Arbor, Michigan, 1995.

[2] D. Witters and C. Campbell, Wirless Medical Telemetry: Addressing the Interference Issue. Jospeh Dyro, Clinical Engineering Handbook, Burlington, MA: Elsevier Press, 2004. pp 492-497.

[3] MUSE, Cardiology Information System Manual, General Electric Company, 2008. 